\title{
BMJ Open Scoping review protocol: bladder cancer in Nigeria: what are the gaps in clinical care and research?
}

\author{
Joyce Kibaru (D) , ${ }^{1}$ Pinky Kotecha, ${ }^{1}$ Abdulkarim Muhammad lya,${ }^{2}$ Beth Russell, ${ }^{1}$ \\ Muzzammil Abdullahi, ${ }^{2}$ Sani Usman Alhassan, ${ }^{2}$ Muhammad Inuwa Mustapha, ${ }^{3}$ \\ Richard T Bryan, ${ }^{4}$ Mieke Van Hemelrijck (D) ${ }^{1}$
}

To cite: Kibaru J, Kotecha P, lya AM, et al. Scoping review protocol: bladder cancer in Nigeria: what are the gaps in clinical care and research? BMJ Open 2021;11:e041894. doi:10.1136/ bmjopen-2020-041894

- Prepublication history and additional material for this paper is available online. To view these files, please visit the journal online (http://dx.doi.org/10. 1136/bmjopen-2020-041894).

Received 19 June 2020 Revised 06 January 2021 Accepted 07 January 2021

Check for updates

(C) Author(s) (or their employer(s)) 2021. Re-use permitted under CC BY-NC. No commercial re-use. See rights and permissions. Published by BMJ.

${ }^{1}$ Faculty of Life Sciences and Medicine, Translational Oncology and Urology Research, King's College London, London, UK ${ }^{2}$ Urology Unit, Department of Surgery, Aminu Kano Teaching Hospital, Kano, Nigeria ${ }^{3}$ Clinical and Radiation Oncology Unit, Radiology Department, Aminu Kano Teaching Hospital, Kano, Nigeria

${ }^{4}$ Bladder Cancer Group, Institute of Cancer \& Genomic Sciences, University of Birmingham, Birmingham, UK

Correspondence to

Joyce Kibaru;

joyce.w.kibaru@kcl.ac.uk

\section{ABSTRACT}

Introduction Bladder cancer $(\mathrm{BC})$ is the 10th common cancer worldwide and ranks seventh in Nigeria. This scoping review aims to identify the gaps in clinical care and research of BC in Nigeria as part of the development of a larger national research programme aiming to improve outcomes and care of BC.

Methods and analysis This review will be conducted according to Arksey and O'Malley scoping review methodology framework. The following electronic databases will be searched: Medline (using the PubMed interface), Ovid Gateway (Embase and Ovid), Cochrane library and Open Grey literature. Two independent reviewers will screen titles and abstracts and subsequently screen full-text studies for inclusion, any lack of consensus will be discussed with a third reviewer. Any study providing insight into the epidemiology or treatment pathway of BC (RCTs, observations, case series, policy paper) will be included. A data chart will be used to extract relevant data from the included studies. Results will be reported according to the Preferred Reporting Items for Systematic Reviews and Meta-Analyses extension for scoping reviews. A consultation process will be carried out with a multidisciplinary team of Nigerian healthcare professionals, patients and scientists.

Ethics and dissemination The results will be disseminated through peer-reviewed publications. By highlighting the key gaps in the literature, this review can provide direction for future research and clinical guidelines in Nigeria (and other low-income and middle-income countries), where $\mathrm{BC}$ is more prevalent due to local risk factors and healthcare settings.

\section{INTRODUCTION}

Bladder cancer is the 10th most common cancer globally, accounting for $3 \%$ of all new cancer cases and $2 \%$ of all cancer mortalities. These numbers are highest in Southern Europe and lowest in Middle Africa, with age-standardised incidence rates of 26.5 and 1.3 per 100000 , respectively. ${ }^{1}$ The incidence, however, is rising in Africa, with the highest rates recorded in North Africa. A recent review reported an incidence of 10.1 and 5.0 per 100000 in North Africa and sub-Saharan

\section{Strengths and limitations of this study}

- This is a novel scoping review to identify the gaps in clinical care and research of bladder cancer in Nigeria as part of the development of a larger national research programme aiming to improve outcomes and care of bladder cancer.

- This review will extend existing reviews by focusing on all bladder cancer patients, both observational and randomised trial data, and specifically addressing the cultural, societal and clinical context of Nigeria.

- Stakeholders including patients, their family members, urologists, oncologists, physiotherapists will be involved throughout the study.

- The identification and synthesis of data will also cover the grey literature.

- It is possible that our review will not include all articles which have been published in every journal as some may not be accessible.

Africa for males and 2.0 and 1.5 per 100000 for females. ${ }^{2}$ In Nigeria, bladder cancer ranks 7 th overall (2nd in males and 11th in females). ${ }^{3}$

Transitional cell carcinoma (TCG) is the predominant histological subtype of bladder cancer in developed countries. ${ }^{45}$ For example, in the USA, over $90 \%$ of bladder cancers are TCCs. ${ }^{5}$ This type of bladder cancer is associated with smoking ${ }^{6}$ and exposure to chemicals in the dye industry. ${ }^{7}$ It usually presents at an early stage without muscle involvement and is associated with a good prognosis. ${ }^{8}$ Its management depends on the staging; transurethral resection of bladder tumour, cystectomy and intravesical therapy are commonly used in non-muscle invasive bladder cancer, while radical therapy (cystectomy or radiotherapy) with chemotherapy can be used for muscle invasive bladder cancer. ${ }^{9}$ In addition to TCC, bladder cancer can also present as squamous cell carcinoma (SCC). 
SCC has two subtypes, schistosomiasis (Bilharzia) associated and non-schistosomiasis associated SCC. Schistosomiasis associated SCC is common in regions where Schistosoma haematobium infection is endemic, such as the Middle East and parts of Africa. Mechanisms through which schistosomiasis lead to bladder cancer include inducing irritation and inflammation ${ }^{10}{ }^{11}$ and chronic bacterial infections. ${ }^{12}$ In contrast to TCC, SCC has a poor prognosis with a 5 -year survival rates of $33 \%-48 \% .{ }^{13}$

In Africa, $60 \%$ of bladder cancers cases in Egypt in the 1990's were of SCC subtype ${ }^{14}$; however, SCC cases are now less common in Egypt, representing 20\%-30\% of cases. ${ }^{15}$ SCC was the most frequent subtype of bladder cancer in Northern Nigeria, representing $53 \%$ of cases. ${ }^{16}$ It is interesting to note that despite the lower prevalence of schistosomiasis in Northern Nigeria, ${ }^{17}$ SCC is still the predominant type of bladder cancer Nigeria. ${ }^{18} 19$ This highlights the possible role of a yet to be identified environmental and/or genetic factor(s), explaining the disparity in histological type between the northern and southern part of the country.

Currently, there are few studies (epidemiological, clinical and translational) on bladder cancer in Nigeria. Hence, this scoping review aims to identify gaps in clinical care and research on bladder cancer in Nigeria.

\section{Study rationale}

Despite bladder cancer being the seventh most common cancer in Nigeria, to our knowledge, there are no national guidelines for its diagnostic and treatment pathways. This scoping review therefore forms part of the development of a national bladder cancer research programme in Nigeria, with an overall aim of improving the outcomes and care of bladder cancer.

\section{Study objectives}

This scoping review is set out to identify the current gaps in knowledge on epidemiology and clinical care of bladder cancer in Nigeria, with an ultimate aim of guiding future research and guideline development as to improve the quality of life and survival of those diagnosed with bladder cancer in Nigeria-and potentially subSaharan Africa and other low-income and middle-income countries.

\section{Protocol design}

A scoping review methodology was chosen to map the literature and identify key concepts and gaps in the research. The methodology for this scoping review was developed based on the Joanna Briggs Institute guidelines $^{20}$ and more specifically the framework developed by Arksey and O'Malley. ${ }^{21}$ Preferred Reporting Items for Systematic Reviews and Meta-Analyses extension for scoping reviews ${ }^{22}$ will be followed to ensure all suggested items are reported. Our six stages methodology framework is outlined below. Our protocol is an adaption of our previously published protocol for a scoping review on the role of physical activity in the management of bladder cancer. $^{23}$

\section{Patient and public involvement}

As the scoping review methodology is based on reviewing and collecting data from publicly available materials, there will be no patient and public involvement. However, in the consultation phase is part of our Patient and Public Involvement strategy, as we will work actively in partnership with patients, their family members, and healthcare professionals to plan and design a national bladder cancer research strategy. Results of this scoping review will be disseminated through a peer-reviewed publication.

\section{Stage 1: identifying the research question}

The following research question was developed in consultation with the research and clinical teams:

'What is known about the epidemiology and treatment pathway of bladder cancer in Nigeria?'

\section{Stage 2: identifying relevant studies: search strategy}

Nigeria does not have a functional and comprehensive national database; we have, therefore, chosen to review the published literature. We anticipate that publications within this review will be mainly based on single centre studies of bladder cancer experiences. The following databases will be searched from inception until the date in which the searches will be performed (until June 2020): Medline (using the PubMed interface), Ovid Gateway (Embase and Ovid), The Cochrane library and Open Grey literature. The search strategies will be evaluated using the Peer Review of Electronic Search Guidelines. ${ }^{24}$

Search terms have been determined by researching the current available literature and through research input to help guide selection of terms. This was done to ensure they are broad enough to capture any study about bladder cancer in Nigeria. Our scoping review will analyse both quantitative and qualitative data on bladder cancer in Nigeria. To ensure that all relevant information is retrieved, relevant grey literature sources will be searched. The following search terms will be used: 'Bladder cancer.mp AND nigeria.mp'. No further filters or date restrictions will be applied in our search in order to cover as much content as possible. We have included the search terms in online supplemental file 1 .

\section{Stage 3: study selection}

Studies will be considered for inclusion in the scoping review if they provide insight into the epidemiology or treatment pathway of bladder cancer in Nigeria. Studies will be excluded if the publication is not available in English. Case reports will also be excluded in order to reasonably describe epidemiology and treatment patterns. No restrictions will be applied in the context of bladder cancer-the review will cover both transitional and squamous bladder cancer, including all stages (non-muscle invasive, muscle invasive and metastatic). A reference management software (Endnote) will be used to gather all the papers from the digital search process. 
From these references, we will document the exclusion process of studies, first based on the title and then based on abstracts. Two review authors will screen the studies independently and any lack of consensus will be discussed with a third review author. After screening titles and abstracts, two reviewers will read the full article and consider it for the review. Those articles excluded will have recorded evidence as to why this was necessary and if there is a lack of consensus, this will be discussed with a third review author. For those studies with multiple publications of the same outcome, the one with the longest follow-up will be selected.

\section{Stage 4: charting the data}

Two independent reviewers will extract the data into a data extraction table to capture the following information:

- Author.

- Year of publication.

- Aims/purpose.

- Study population and sample size-including bladder cancer type and treatment where available.

- Study design.

- Intervention type, comparator and details.

- Outcomes (eg, long-term survival and cancer-free survival).

- Key findings that relate to the objectives.

\section{Stage 5: collating, summarising and reporting the results}

A descriptive numerical summary and a thematic analysis will be performed on the studies analysed-whereby we will aim to map all findings and gaps across the bladder cancer treatment pathway, from prevention to diagnosis, management, survivorship and palliative care. Descriptive numerical summary will include information on characteristics of included studies such as: the number of studies included, study designs, years of publication, intervention types and study populations.

Study information will thus be summarised in themes in order to highlight the current state of understanding of epidemiology and treatment pathway of bladder cancer (eg, risk factors, descriptive epidemiology, diagnostic pathway, various treatment pathways for different stages of bladder cancer, outcomes, survivorship). Gaps in clinical research and care will be identified. Recommendations will be given to guide direction in improving bladder cancer research.

Given the nature of this scoping review, we will not be explicitly performing a risk of bias assessment as usually required for quantitative systematic reviews ${ }^{22}$ for the following four reasons: (1) We will not compare the clinical outcomes of any clinical studies, but only describe interventions used and their potential effects; (2) argumentative and qualitative data do not lend themselves to the risk of bias assessment common in quantitative systematic reviews; (3) we are likely to include many different types of studies which evaluations would have to be type-specific and (4) quality assessments are likely be very difficult to standardise between reviewers and which could potentially add unnecessary bias into our own analyses.

\section{Stage 6: consultation: patient and public involvement}

This scoping review is a first phase in developing a national bladder cancer research programme in Nigeria. To ensure that our assessment of the existing evidence for epidemiology and treatment pathways of bladder cancer reflects the current practice in Nigeria, we also aim to include a consultation phase in this scoping review. The results from this scoping review combined with the consultation phase will then lead to development of a research strategy to further improve the clinical care of bladder cancer patients.

This consultation phase is part of our patient and public involvement strategy as we will work actively in partnership with patients, their family members and healthcare professionals to plan and design a national bladder cancer research strategy. More specifically, we will run focus groups with a multidisciplinary team in Nigeria consisting of urologists, oncologists, histopathologists, epidemiologists and molecular biologists.

\section{Ethics and dissemination}

As outlined above, this scoping review with a consultation phase will constitute the first stage of our development of a national bladder cancer research programme in Nigeria. Results will be disseminated through a peerreviewed publication. Through the consultation step, we will ensure that findings will reach a wide audience and recommendations can be made for future development of clinical bladder cancer research studies.

As the scoping review methodology is based on reviewing and collecting data from publicly available materials, this study does not require ethics approval. This consultation step will be carried out as part of patient and public involvement, so this study does not require ethical review.

\section{Twitter Muhammad Inuwa Mustapha @mohdimustapha}

Acknowledgements The authors gratefully acknowledge the helpful comments from the bladder cancer patient support group.

Contributors Design of protocol: JK, PK, AMI and MVH. Draft of manuscript: JK, PK, AMI and BR. Final approval of manuscript: JK, PK, AMI, BR, MA, SUA, MIM, RTB and MVH.

Funding The authors have not declared a specific grant for this research from any funding agency in the public, commercial or not-for-profit sectors.

Competing interests None declared.

Patient consent for publication Not required.

Provenance and peer review Not commissioned; externally peer reviewed.

Supplemental material This content has been supplied by the author(s). It has not been vetted by BMJ Publishing Group Limited (BMJ) and may not have been peer-reviewed. Any opinions or recommendations discussed are solely those of the author(s) and are not endorsed by BMJ. BMJ disclaims all liability and responsibility arising from any reliance placed on the content. Where the content includes any translated material, BMJ does not warrant the accuracy and reliability of the translations (including but not limited to local regulations, clinical guidelines, terminology, drug names and drug dosages), and is not responsible for any error and/or omissions arising from translation and adaptation or otherwise. 
Open access This is an open access article distributed in accordance with the Creative Commons Attribution Non Commercial (CC BY-NC 4.0) license, which permits others to distribute, remix, adapt, build upon this work non-commercially, and license their derivative works on different terms, provided the original work is properly cited, appropriate credit is given, any changes made indicated, and the use is non-commercial. See: http://creativecommons.org/licenses/by-nc/4.0/.

\section{ORCID iDs}

Joyce Kibaru http://orcid.org/0000-0002-9356-0810

Mieke Van Hemelrijck http://orcid.org/0000-0002-7317-0858

\section{REFERENCES}

1 Bray F, Ferlay J, Soerjomataram I, et al. Global cancer statistics 2018: GLOBOCAN estimates of incidence and mortality worldwide for 36 cancers in 185 countries. CA Cancer J Clin 2018;68:394-424.

2 Adeloye D, Harhay MO, Ayepola OO, et al. Estimate of the incidence of bladder cancer in Africa: a systematic review and Bayesian metaanalysis. Int J Urol 2019;26:102-12.

3 Mohammed AZ, Edino ST, Ochicha O, et al. Cancer in Nigeria: a 10-year analysis of the Kano cancer registry. Niger J Med 2008;17:280-4.

4 Chavan S, Bray F, Lortet-Tieulent J, et al. International variations in bladder cancer incidence and mortality. Eur Urol 2014;66:59-73.

5 Lamm DL, Torti FM. Bladder cancer, 1996. CA Cancer J Clin 1996;46:93-112.

6 Colombel M, Soloway M, Akaza H, et al. Epidemiology, staging, grading, and risk stratification of bladder cancer. European Urology Supplements 2008;7:618-26.

7 Case RA, Hosker ME, McDonald DB, et al. Tumours of the urinary bladder in workmen engaged in the manufacture and use of certain dyestuff intermediates in the British chemical industry. I. The role of aniline, benzidine, alpha-naphthylamine, and beta-naphthylamine. $\mathrm{Br}$ $J$ Ind Med 1954;11:75-104.

8 Bowa K, Mulele C, Kachimba J, et al. A review of bladder cancer in sub-Saharan Africa: a different disease, with a distinct presentation, assessment, and treatment. Ann Afr Med 2018;17:99-105.

9 National Institute for Health and Care Excellence. Bladder cancer: diagnosis and management NICE guideline [NG2], 2015. Available: https://www.nice.org.uk/guidance/ng2
10 Rosin MP, Anwar WA, Ward AJ. Inflammation, chromosomal instability, and cancer: the schistosomiasis model. Cancer Res 1994;54:1929s-33.

11 Rosin MP, Saad el Din Zaki S, Ward AJ, et al. Involvement of inflammatory reactions and elevated cell proliferation in the development of bladder cancer in schistosomiasis patients. Mutat Res 1994;305:283-92.

12 Mostafa MH, Sheweita SA, O'Connor PJ. Relationship between schistosomiasis and bladder cancer. Clin Microbiol Rev 1999;12:97-111.

13 Manunta A, Vincendeau S, Kiriakou G, et al. Non-transitional cell bladder carcinomas. BJU Int 2005;95:497-502.

14 Ghoneim MA, el-Mekresh MM, el-Baz MA, et al. Radical cystectomy for carcinoma of the bladder: critical evaluation of the results in 1,026 cases. J Urol 1997:158:393-9.

15 Felix AS, Soliman AS, Khaled H, et al. The changing patterns of bladder cancer in Egypt over the past 26 years. Cancer Causes Control 2008;19:421-9.

16 Eni HNa U, Nggada H, Dogo D. Carcinoma of the urinary bladder in Maiduguri: the schistosomiasis connection. Int J Oncol 2007;5.

17 arcgis. Global prevalence of STh and schistosomiasis, 2020. Available: https://lshtm.maps.arcgis.com/apps/webappviewer/index. $\mathrm{html}$ ?id=2e1bc70731114537a8504e3260b6fbc0

18 Ochicha O, Alhassan S, Mohammed AZ, et al. Bladder cancer in Kano--a histopathological review. West Afr J Med 2003;22:202-4

19 Mungadi IA, Malami SA. Urinary bladder cancer and schistosomiasis in north-western Nigeria. West Afr J Med 2007;26:226-9.

20 Peters MDJ, Godfrey C, McInerney P. Chapter 11: scoping reviews (2020 version). In: Aromataris E, Munn Z, eds. Joanna briggs institute reviewer's manual. Adelaide: JBI, 2020. https://reviewersmanual. joannabriggs.org/

21 Arksey H, O'Malley L. Scoping studies: towards a methodological framework. Int J Soc Res Methodol 2005;8:19-32.

22 Tricco AC, Lillie E, Zarin W, et al. PRISMA extension for scoping reviews (PRISMA-ScR): checklist and explanation. Ann Intern Med 2018;169:467-73.

23 Mehrotra S, Rowland M, Zhang $\mathrm{H}$, et al. Scoping review protocol: is there a role for physical activity interventions in the treatment pathway of bladder cancer? BMJ Open 2019;9:e033518.

24 McGowan J, Sampson M, Salzwedel DM, et al. PRESS Peer Review of Electronic Search Strategies: 2015 Guideline Statement. J Clin Epidemiol 2016;75:40-6. 